\title{
Measuring Nightmare Frequency: Retrospective Questionnaires Versus Prospective Logs
}

\author{
J. Lancee*,1, V.I. Spoormaker ${ }^{2}$, G. Peterse ${ }^{1}$ and J. van den Bout ${ }^{1}$ \\ ${ }^{1}$ Utrecht University, Clinical and Health Psychology, PO Box 80.140, 3508 TC, Utrecht, The Netherlands \\ ${ }^{2}$ Max Planck Institute of Psychiatry, Kraepelinstr. 2-10, 80804, Munchen, Germany
}

\begin{abstract}
Retrospective measurements underestimate nightmare frequency, but little is known about how retrospective duration and attention for nightmares may affect this process. This study evaluates the differences between two retrospective durations, a prospective log, and a retrospective estimate after keeping this log. Forty-nine participants completed the SLEEP-50, kept a nightmare log, and estimated their nightmares after keeping a log. Paired t-tests showed that estimates of nightmare frequencies differed significantly from each other according to measurement type $(\mathrm{p}<.05)$. Prospective logs are the most accurate way of estimating nightmare frequency, possibly due to simply forgetting over time. For treatment studies relying solely on retrospective measurements, a short duration is recommended.
\end{abstract}

Keywords: Nightmare frequency, retrospective estimate, prospective log.

\section{INTRODUCTION}

About $2-5 \%$ of the general population has frequent nightmares [1-4]. Nightmares have a negative effect on sleep [5], and are associated with mental complaints [6-9]. Nightmares are usually measured by prospective logs or retrospective questionnaires. Although not objective, these measurements can distinguish between nightmare frequency and nightmare distress, two related but different concepts [10].

Several authors have noted that retrospective questionnaires tend to underestimate nightmare frequency compared to prospective logs $[6,11,12]$. Wood and Bootzin [11] tested this discrepancy and found significant differences $(p<.01)$ between prospective and retrospective measurements. No statistical differences $(\mathrm{p}>.05)$ were found between monthly or yearly retrospective estimates in two studies $[6,11]$. Both these studies however, used undergraduates of whom high percentages had no nightmares at all.

Schredl et al. [13] argue that prospective logs provide more accurate data because there is less chance of forgetting over time, and the attention of the respondents is better focused on their nightmares. Other authors have hypothesized that the underestimation in retrospective estimates may be due to a motivated forgetting caused by the negative emotions of nightmares [6]. In effect-studies, however, retrospective questionnaires are still commonly used because 1) they are easy to complete and 2) persons may be reluctant to keeping a log; thus limiting the already high dropout-rates in nightmare treatment studies $[6,14]$.

The aim of this study is to determine the differences between prospective and retrospective measurements, and to evaluate if a short duration questionnaire (e.g., one week) is a more reliable alternative to prospective $\operatorname{logs}$ than longer duration questionnaires (e.g., one month). Moreover, to test if keeping a log increases focus of attention on nightmares,

*Address correspondence to this author at the Department of Clinical and Health Psychology, PO Box 80.140, 3508 TC, Utrecht, Utrecht University, The Netherlands; E-mail: J.lancee@uu.nl we evaluated whether the retrospective estimate would be increased after keeping a $\log$.

\section{MATERIALS AND METHODOLOGY}

\section{Participants}

Seventy-seven psychology students filled in the retrospective questionnaire, 58 of whom completed a two-week nightmare diary. A total of 49 further estimated their number of nightmares after the diary period. Of the final sample $93.9 \%$ (46) were female. Mean age was $22.8(\mathrm{SD}=3.3)$.

\section{Measurements}

For the assessment of retrospective nightmare frequency the SLEEP-50 [15] was used. The SLEEP-50 has good reliability (Cronbach's alpha $=.85$, test retest reliability $.65-$ .89 ), and validity outcomes. For nightmares, the sensitivity (proportion correctly diagnosed with nightmares according to the DSM-IV) was .84 and the specificity (proportion correctly classified without) was .77 . A nightmare diary measured prospective nightmare frequency. The diary was kept for fourteen days. Participants were asked each consecutive day if they had a nightmare, whether they woke up from this nightmare, and how intense this dream was.

\section{Procedure}

Participants were psychology students who were recruited at a digital learning environment at Utrecht University. Only people with nightmares could apply. Participants filled out a retrospective questionnaire in which they had to estimate their nightmare frequency per month (month) and their nightmare frequency in the last week (week). After completion, a two week-nightmare diary was sent to their home address (log). After handing in the diary, participants were asked to estimate their nightmare frequency for the diary period (week-after-log). Participants received course credits for participation.

\section{Statistical Analysis}

Differences were measured with paired t-tests (1-tailed), with a significance level of $\mathrm{p}=.05$. 


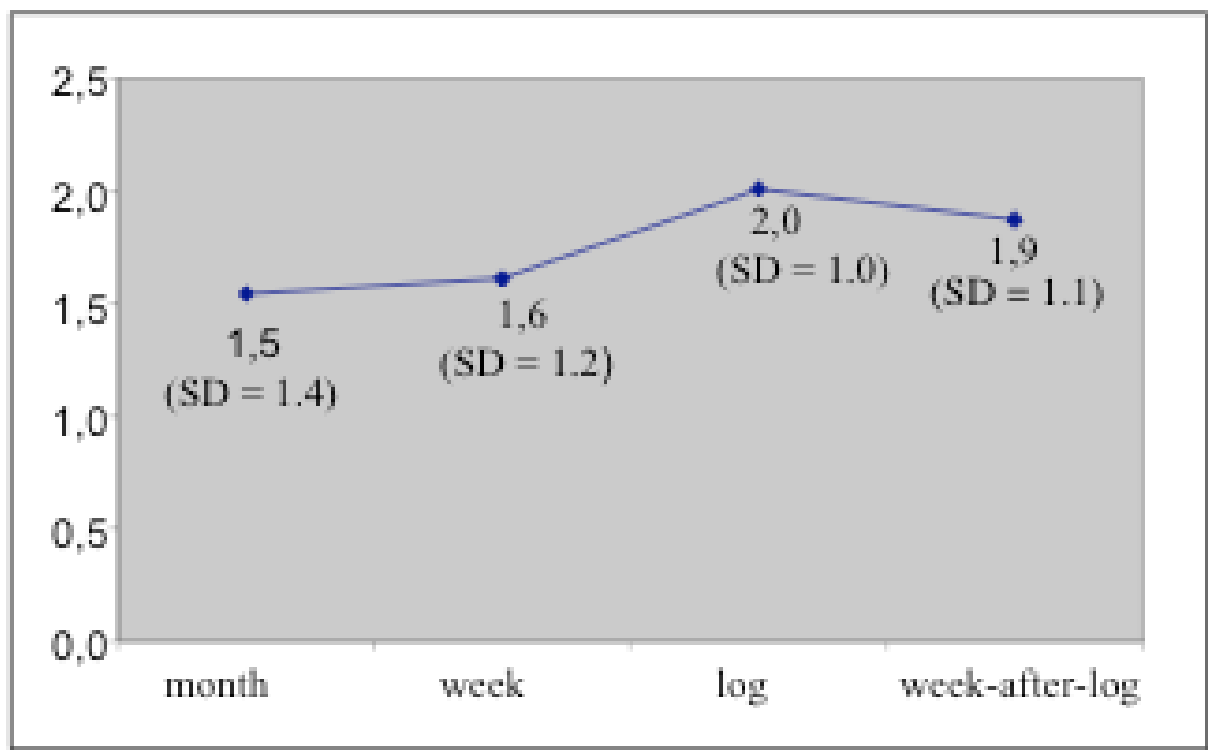

Fig. (1). Nightmare frequency transformed to a one week period.

Table1. Paired t-tests for all the Different Types of Nightmares Frequency Transformed to a One Week Period

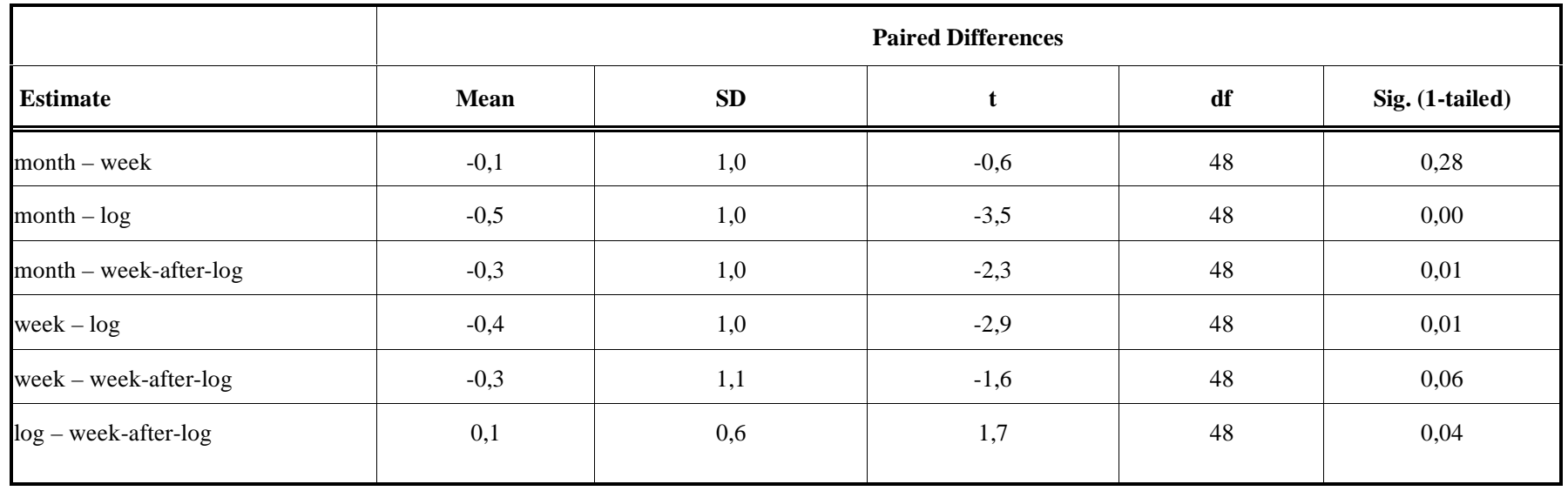

\section{RESULTS}

Paired t-tests showed that most of the frequencies differed significantly from each other (see Table 1), with the exception of the retrospective week- versus month-estimate $(\mathrm{t}(48)=-0.1, \mathrm{p}=.28)$. However, this difference was significant when the test was conducted on all 77 participants who filled out both, $\mathrm{t}(76)=-2.0, \mathrm{p}<.05)$.

\section{DISCUSSION}

In accordance to Wood and Bootzin [11] this study found a significant discrepancy between retrospective and prospective measurements. Prospective logs are a more accurate way of assessing nightmare frequency. Retrospective questionnaires underestimate nightmare frequency, but the shorter the duration the more accurate the estimate, a finding relevant for treatment studies. This may be an attention issue - participants may simply forget nightmares over time [13]. In line with this explanation, this study showed that keeping a nightmare log led to more accurate retrospective estimates.

Although the current study had a limited sample size, an a priori power calculation showed that current numbers were sufficient to obtain significant differences, such as found in this study. Future studies aiming to compare different alternatives for assessing nightmare frequency could include a yearly estimate and a retrospective estimate some time after a log has been kept. Moreover, it would be interesting to evaluate more objective forms of sleep assessment. Although polysomnography has been shown to reduce nightmare frequency [16], actigraphy may be less intrusive.

Nightmare studies should by default use prospective measurements or, in case of treatment studies, retrospective questionnaires with the shortest duration possible.

\section{REFERENCES}

[1] Stepansky R, Holzinger B, Schmeiser-Rieder A, Saletu B, Kunze M, Zeitlhofer J. Austrian dream behavior: results of a representative population survey. Dreaming 1998; 8: 23-31.

[2] Hublin C, Kaprio J, Partinen M, Koskenvuo M. Nightmares: familial aggregation and association with psychiatric disorders in nationwide twin cohort. Am J Med Genet 1999; 88: 329-336.

[3] Klink M, Quan S. Prevalence of reported sleep disturbances in a general population and their relation to obstructive airway disease. Chest 1987; 91: 540-546. 
[4] Spoormaker VI, van den Bout J. The prevalence of sleep disorders in the Netherlands. Sleep-Wake Res Netherlands 2005; 16: 155158.

[5] Kales A, Soldatos C, Caldwell AB, et al. Nightmares: clinical characteristics and personality patterns. Am J Psychiat 1980; 137: 1197- 1201.

[6] Zadra AL, Donderi DO. Nightmares and bad dreams: their prevalence and relationship to well-being. J Abnorm Psychol 2000; 109: 273-281.

[7] Köthe M, Pietrowsky R. Behavioral effects of nightmares and their correlations to personality patterns. Dreaming 2001; 11: 43-52.

[8] Berquier A, Ashton R. Characteristic of the frequent nightmare sufferer. J Abnorm Psychol 1992; 101: 246-250.

[9] Levin R, Fireman G. Nightmare prevalence, nightmare distress, and self reported psychological disturbance. Sleep 2002; 25: 205-212.

[10] Belicki K. Nightmare frequency versus nightmare distress: relations to psychopathology and cognitive style. J Abnorm Psychol 1992; 101: 592-597.
[11] Wood JM, Bootzin RR. The prevalence of nightmares and their independence from anxiety. J Abnorm Psychol 1990; 99: 64-68.

[12] Blagrove M, Farmer L, Williams E. The relationship of nightmare frequency and nightmare distress to well-being. J Sleep Res 2004; 13: $129-136$

[13] Schredl M. Questionnaires and diaries as research instrument in dream research: methodological issues. Dreaming 2002; 12: 17-26.

[14] Neidhardt EJ, Krakow B, Kellner R, Pathak D. The beneficial effects of one treatment session and recording of nightmares on chronic nightmare sufferers. Sleep 1992; 15: 470-473.

[15] Spoormaker VI, Verbeek I, van den Bout J, Klip EC. Initial validation of the SLEEP-50 questionnaire. Behav Sleep Med 2005; 3 : 227-246.

[16] Spoormaker VI, Schreuder JN, de Weerd AW, Kleijn WC, Kamphuisen HAC. Polysomnography reduces nightmare frequency. Sleep-Wake Res in the Netherlands 2006; 17: 113-116. 